\title{
Simultaneous Measurement of Particle Size and Electrostatic Charge by using the Laser Doppler Method $\dagger$
}

\author{
Noriyoshi Kaya, Hiroyuki Tsujimoto, Shuji Sasabe, \\ Department of Technological Development, Hosokawa Micron Co., Ltd. . \\ Shinchi Yoshikawa and M.K. Mazumder \\ Univ. of Arkansas at Little Rock, College of Sci. and Eng. Technology **
}

\section{Introduction}

The electrostatic photography using toner particles is widely used by copiers, facsimile machines and laser printers. In the electrostatic development process, the electrostatic characteristics of toner particles have been extensively studied and measured due to their importance in determining the quality of finished print.

In the field of aerosols, on the other hand, since the behavior of aerosol particle also depends highly on the electrostatisticity of particles, many methods for measuring electrostatic charges have been studied. One of our authors proposed that the particle size and charge can be measured by applying one of the methods employed in the field of aerosols to toner particles ${ }^{1)}$. When the toner particles are introduced into a detection area with electric and acoustic field simultaneously formed therein, the velocity of the particle is measured by the Laser Doppler method, and the particle size and electrostatic charge are calculated from the measured data ${ }^{2}$. The measurements of toner particles have confirmed the proposal and an instrument applied this method has been devised.

In this paper, this method of measurement is explained, and presented some applied examples for evaluating of the electrostatic characteristics of toner particles, as well as examples of recent measurements of mono-component toners with a modified toner feeding apparatus.

\section{Principle of measurement}

When charged particles vertically fall through acoustic and electric fields, as shown in Figure 1, the horizontal behavior of the particles is expressed by:

* 1-9 Shoudai-Tajika, Hirakata, Osaka 573 Japan

** ETAS 575-2801 South Univ., Little Rock Arkansas 722204 USA

$\dagger$ This report was originally printed in J. Soc. Powder Technology, Japan. 31, 202 (1994) in Japanese, before being translated into English with the permission of the editorial committee of the Soc. Powder Technology, Japan.

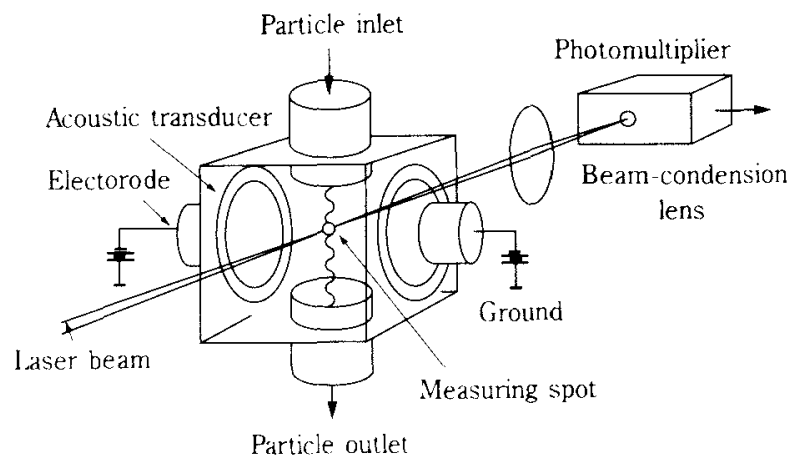

Fig. 1 Behavior of a particle subjected to acoustic and electric fields in a measurement cell

$$
m_{p} \frac{d \nu_{p}}{d_{t}}+\frac{3 \pi \eta D_{p}}{C_{m}}\left(\nu_{p}-u_{g} \sin \omega t\right)=q E
$$

where $m_{p}$ : mass of particle $(\mathrm{kg}), v_{p}$ : velocity of particle $(\mathrm{m} / \mathrm{s}), \eta$ : viscosity of air $(\mathrm{Pa} \cdot \mathrm{s}), \omega$ : angular velocity of sound $(\mathrm{rad} / \mathrm{s}), D_{p}$ : particle size $(\mathrm{m}), u_{g}$ :amplitude of sound $(\mathrm{m}), \mathrm{C}_{\mathrm{m}}$ : Cunningham's correction factor, $q$ : charge of particle $(\mathrm{C}), E$ : electric field strength $(\mathrm{V} / \mathrm{m})$. By solving Eq. (1) at $\tau_{p}$ : relaxation time of particle $=m_{p} \mathrm{C}_{\mathrm{m}} / 3 \pi \eta D_{p}$, generally, we obtain

$$
\begin{aligned}
\nu_{p} & =\frac{u_{g}}{\sqrt{\left(1+\tau_{p}^{2} \omega^{2}\right)}} \sin (\omega t-\phi) \\
& +\exp \left(-\frac{t}{\tau_{p}}\right) \sin \phi+k q E\left\{1-\exp \left(-\frac{t}{\tau_{p}}\right)\right\}(2)
\end{aligned}
$$

where $\phi$ is the angle of phase lag (rad), and enters in the following relation with the relaxation time $\tau_{p}(\mathrm{~s})$.

$$
\tau_{p} \omega=\tan \phi \quad D_{p}=\left(\frac{18 \eta \tan \phi}{\rho_{\rho} C_{m} \omega}\right)^{0.5}
$$

In the above formula, $\rho_{\rho}$ is the density of particles $\left(\mathrm{kg} / \mathrm{m}^{3}\right), \mathrm{k}=\mathrm{C}_{\mathrm{m}} / 3 \pi \eta D_{p}$. Thus, the particle size $D_{p}$ (m) can be obtained from Eq. (3), if the phase 
$\operatorname{lag} \phi$ caused by a reference sound signal and actual vibration of the particle is known.In Eq. (3), at $\mathrm{C}_{\mathrm{m}}=$ $1.0, \varrho_{p}=1000\left(\mathrm{~kg} / \mathrm{m}^{3}\right), D_{p}$ is generally an aerodynamic particle size $D_{p a}$.

As Cunningham's correction factor $\mathrm{C}_{\mathrm{m}}$ becomes $\mathrm{C}_{\mathrm{m}}$ $\fallingdotseq 1$, where particle size is larger than $1.2 \mu \mathrm{m}$ or more, most of the toner particles with a diameter less than $25 \mu \mathrm{m}$ are calculated using $C_{m}=1$. In the Laser Doppler method ${ }^{3)}$, a beat frequency $\Delta f(\mathrm{~Hz})$ generated by a laser beams applied from two directions to an object moving at a velocity $v(\mathrm{~m} / \mathrm{s})$ is

$$
\nabla f=\frac{2 v \sin \theta \cos \psi}{\lambda}
$$

where $\theta$ : half crossing angle of laser beams (rad), $\lambda$ : wave length of laser $(\mathrm{m}), \psi$ : inclination of movement of particle in the direction of two crossing beams (rad). In the apparatus, since the inclination $\psi$ seem to be that $p_{s i}=0$, and the crossing angle $2 \theta$ and wavelength $\lambda$ of the laser beams are constant, when the position of optical elements are fixed, from Eq. (4), the beat frequency $\Delta f$ is proportional to the velocity of particles $v_{p}$ reduced from Eq. (4).

Separating the particle velocity $v_{p}$ into 2 velocity components, $v_{a}$ and $v_{e}$, from Eq. (2). The $v_{a}$ is a particle velocity component into the acoustic field in the steady state and the $v_{e}$ is the moving velocity in the electric field formed by parallel plate electrodes with an applied voltage $V$, and the distance between the plates being $L$.

Thus the beat frequencies $\Delta f_{a}$ and $\Delta f_{e}$ are respectively defined by,

$$
\begin{aligned}
& \Delta f_{a} x \frac{u_{g}}{\sqrt{\left(1+\tau_{p}^{2} \omega^{2}\right)}} \sin (\omega t-\phi) \\
& \Delta f_{e} x \frac{C_{m}}{3 \pi \eta D_{p}} q\left(\frac{V}{L}\right)
\end{aligned}
$$

A beat frequency actually measured at the velocity $v_{p}$ of particles is a combination of Eqs. (5) and (6), a signal with an offset $\phi$ in relation to an acoustic reference signal is obtained by subjecting the beat frequency to FM demodulation at a frequency of acoustic oscillation, and the particles size $D_{p}$ can be obtained by using Eq. (3). Regarding the quantity of charge, the velocity $v_{e}$ of the movement is obtained by the beat frequency counted, and then input into a computer along with the particle size $D_{p}$ and voltage $V$ applied to the electrodes, and the quantity $q$ of charge of the particles can be obtained by calculating Eq. (6).

\section{Measuring apparatus}

An optical measurement system and signal process-

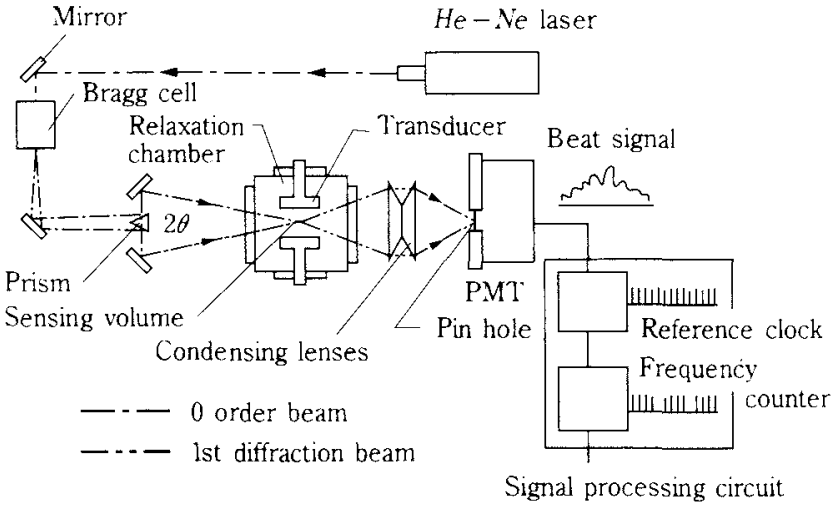

Fig. 2 Optical system and signal processing by the laser Doppler method

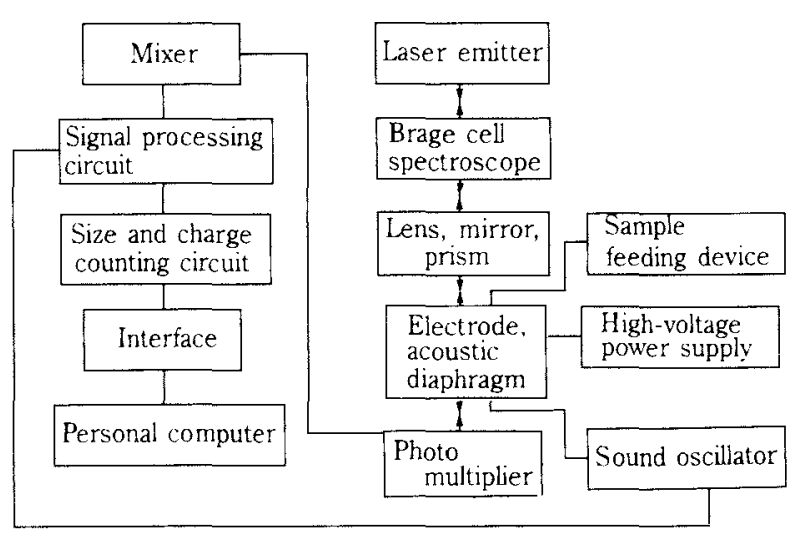

Fig. 3 Block diagram of measurement apparatus

ing by the laser Doppler method are explained in Figure 2, and a block diagram of the entire apparatus is shown in Figure 3.

A speaker acting as an electrode is attached to the side surface of the measurement cell, and acoustic oscillation of $1 \mathrm{kHz}$ is used. The electrode on the opposite side is given an opposite polarity, so that the positive and negative voltages are applied evenly to the grounded frame of the apparatus.

Test particles introduced through a sample hole fall downward into the measurement cell, where they are shifted by the acoustic and electric fields. A laser beam is split by a Bragg Cell and the two resulting beams are then modulated to a frequency of $41 \mathrm{MHz}$, later intersecting each other in the center of a detecting area at a crossing angle of $6^{\circ}$. Here, the velocity of the particle shifted by the electrodes and acoustic oscillation is measured by a photomultiplier using the Laser Doppler method. The signal includes a beat frequency $\Delta f$ proportional to the velocity $v_{p}$, and is electrically measured by separating the oscillating 
velocitan of the particles induced by acoustic oscillation from the velocity of movement induced by electric charge. From Eq. (5), the beat frequency $\Delta f_{a}$ of the particles induced by acoustic oscillation shows a fluctuating sine wave at a frequency of $1 \mathrm{kHz}$. When the particle is oscillated to a frequency identical to the acoustic oscillation of $1 \mathrm{kHz}$, a periodic signal of sine wave length with a delay corresponding to the phase lag $\phi$ can be obtained. Then, by measuring the phase lag, the particle size can be obtained. Additionally, the beat frequency $\Delta f_{e}$ caused by the movement of the particles due to electrostatic charging corresponds to a deviation from $41 \mathrm{MHz}$, and the velocity $v_{p}$ of the movement is obtained by measuring the frequency. Then the particle size $D_{p}$ and quantity of charge $q$ can be obtained from Eqs. (5) and (6). In Figures $\mathbf{4}$ and $\mathbf{5}$, a relationship between the particle size and quantity of particle charge can be obtained by applying an electrically simulated signal of the particle to the circuit with a signal generator,

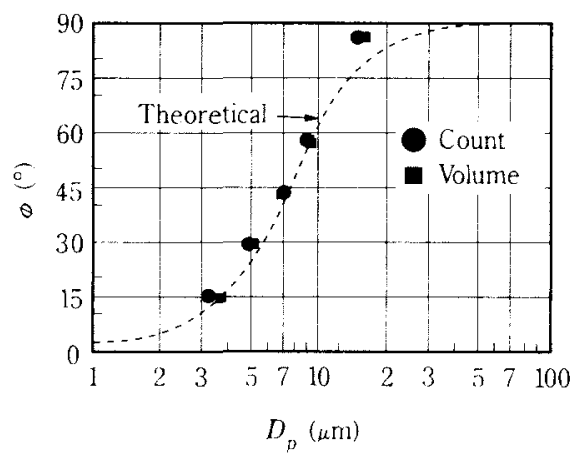

Fig. 4 Relation between phase difference and particle size

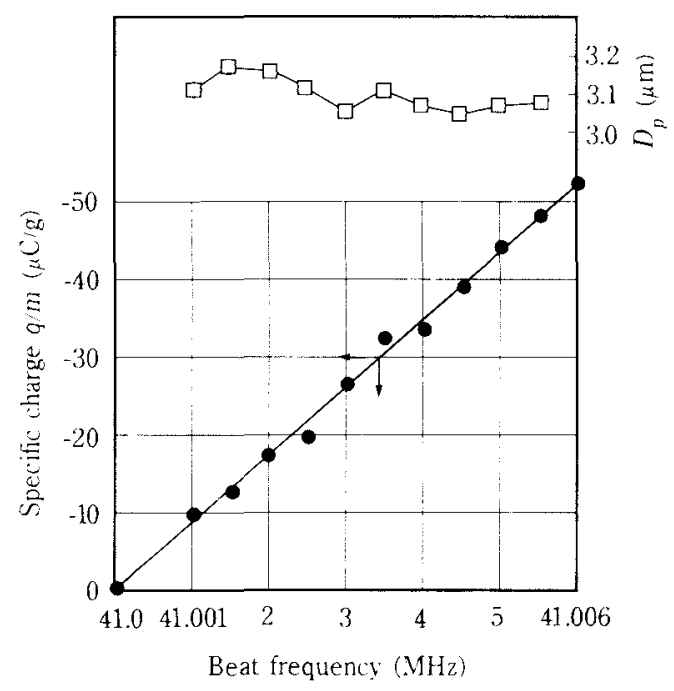

Fig. 5 Relation between quantity of charge and frequency and assuming that the applied voltage is $100 \mathrm{~V}$ for testing the accuracy of the circuit. In Figure 4, a "Theoretical" curve is calculated by Eq. (3) and round and square marks were obtained by applying the simulated signals with a phase delay varied by the signal generator to the circuit. The mean particle size based on the particle count and based on the volume is reffered to as Count and Volume, respectively. Although ideally the mean particle sizes based on the count and volume coincide with each other, and are consistent with the theoretical formula, they do not perfectly coincide due to errors which are derived from the signal generator, measurement circuit and computer.

It is required to charge the test particles before measurement. To achieve the charging, in the case of a two-component toner, the toner is mixed with carrier particles and loaded into the feeding device, where they are subjected to a jet of nitrogen gas. The carrier particles are held in place by an electromagnet, allowing only the toner particles to fall through the measurement area. In the case of a mono-component toner, a feeder utilizing the charging mechanism of copying machines is employed for the measurement as described below. To measure particle charges when using the feeding device, the pressure of the nitrogen gas, angle and position of the nozzle affect the falling direction of the particles. Additionally, the atomospheric conditions of charging the test particles, such as the temperature and humidity in the measurement area, can also significantly affect the measurement. It is very important to consider such conditions with sufficient care.

After entering the measurement area, the particles are affected by the acoustic and electric fields, and biased in the horizontal direction as they fall. Then the beat frequency of the horizontal velocity is measured. In the modified apparatus, the beat frequency measurements are sent to the computer, interrupting any program operating at the time, and the distribution of particle size and the specific particle charge distribution are displayed on the computer screen in real time. After a predetermined number of particles are measured, the screen display is terminated and you can analyze the particle charge characteristics by using a particle size distribution, particle charge distribution and/or a three-dimensional distribution of particle size and charge.

\section{Example of measurement and its evaluation}

An example of a three-dimensional display of a measurement result is shown in Figure 6 . It is 


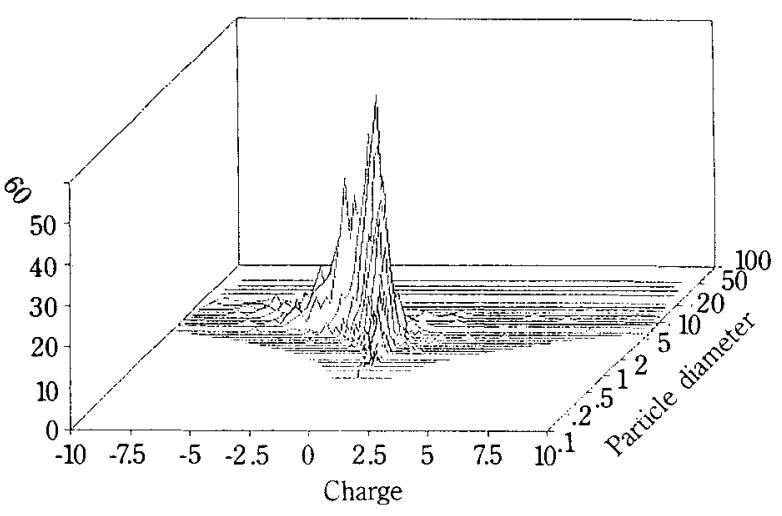

Fig. 6 Three-dimensional representation illustrating simultaneous measurement of quantity of charge and particle size

recognized in the figure that more particles are negatively charged, and the particle sizes range from $1 \mu \mathrm{m}$ to 17 or $18 \mu \mathrm{m}$. Various studies on particle size and charging have been reported by using toner particles up to now ${ }^{4)}$. As an example of these studies, three types of test samples, all having different charges characteristics, are compared in Figure 7 . In the figure, D-1 is negatively charged and D-2 is positively charged, and although these samples have different polarities, both provide the same fine copy quality. It can be observed that the quantity of charge corresponds to the second power of the particle size in test sample D-1 and D-2. However such a relationship can not be observed in test sample D-3, because of poor charge characteristics.

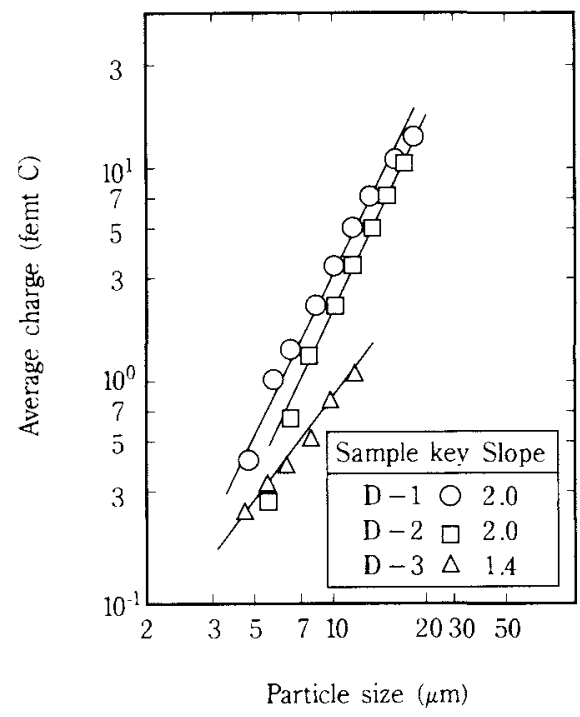

On the right side of Figure 7, the actual quantity of particle charge of the test samples is shown, and it can be seen that the charge quantities are distributed relatively in a higher range.

Figure 8 shows the charge quntities of twocomponent toner in relation to the qualities of the carriers. The same two-component toners were charged by agitating them with two types of carriers (new and used), and measured for evaluating the carrier effects on charging toners. With the used carrier, it is clear that charge quality of toner was generally poor; although some coarse particles have a high charge, the distribution curve is located closer to a neutral point 0 . This may be due to the amount of wear and breakage of toner which occurs during long-term use, and in the event that any toner particles become attached to the carrier surface. This surface contamination can not give sufficient charge to the toner. In fact, we observed, using a microscope, that the used carrier was contaminated by pieces of ground toner fused to its surface. Accordingly, we consider the toner to be insufficiently charged when the carrier surface is contaminated with grounded toner particles because the friction charge level is too low.

Recently, mono-component toners which do not contain carriers, are being used in more and more copying machines and laser printers to reduce the size of the apparatus. This type of toner cannot be charged as easily as a conventional two-component toner and accurate measurement of mono-component toner charges have been difficult. However we have

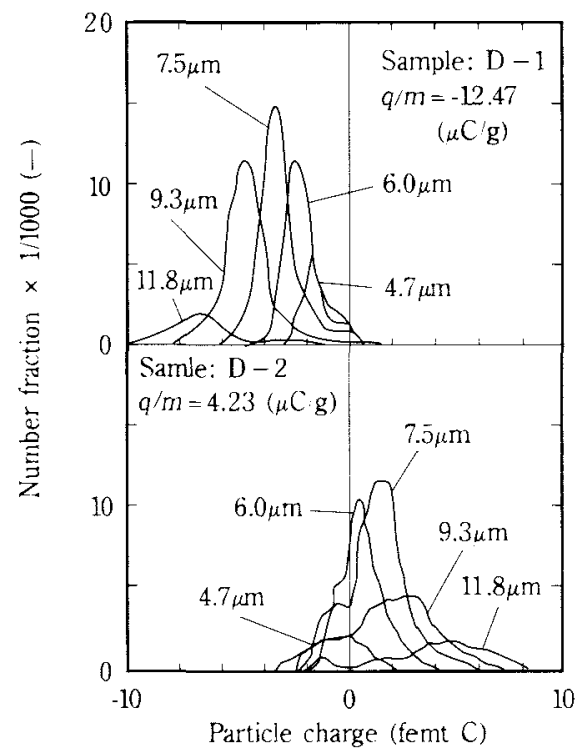

Fig. 7 Comparison of measurement of charge characteristics 

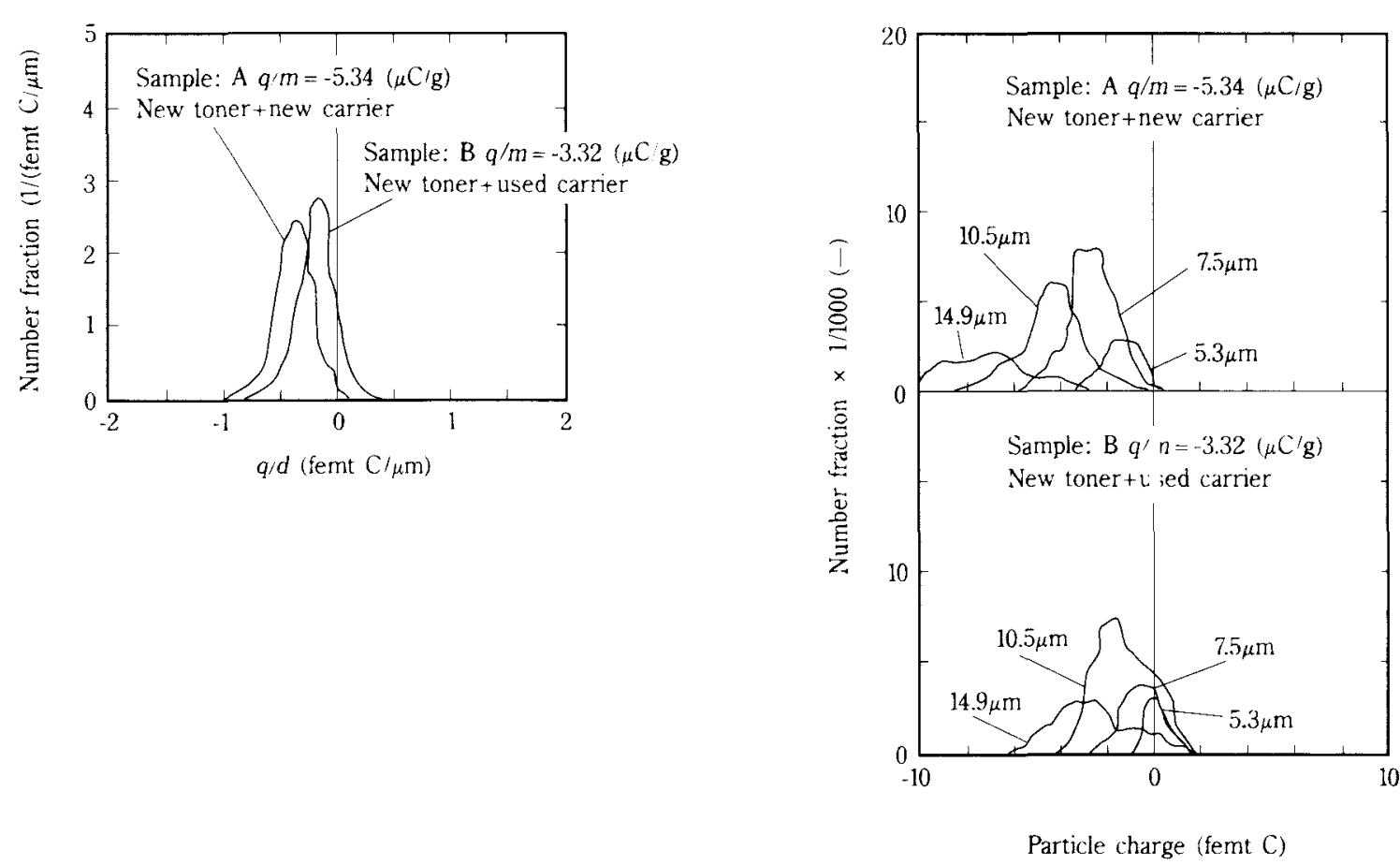

Fig. 8 Effects of new and used carriers on toner
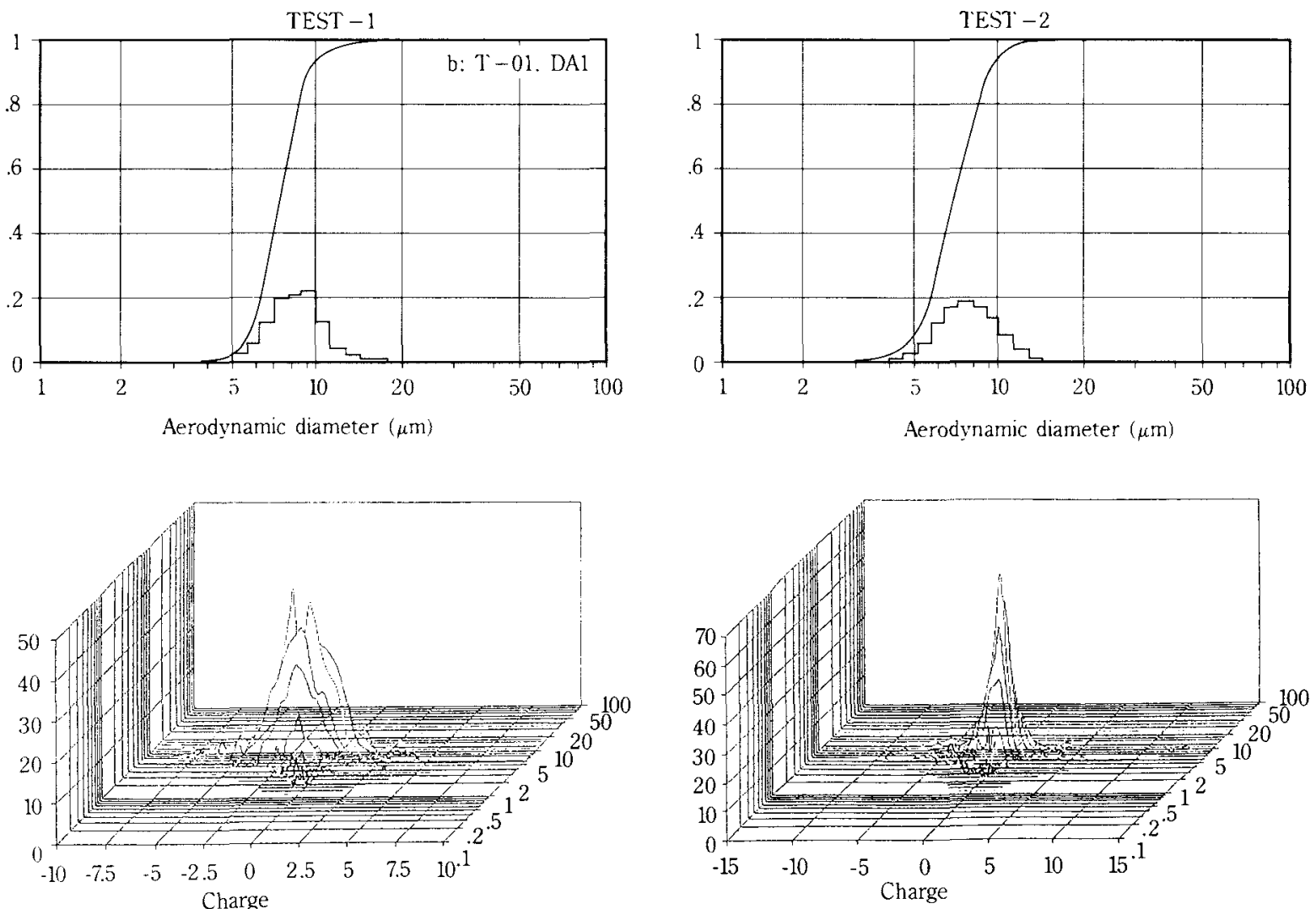

Fig. 9 Comparison example of measurement of single-component toners

recently deviced a method for measuring monocomponent toner charges by physically removing a machine's charging mechanism and adapting it to our feeding device for accurate measurement. An example of a measurement for a mono-component toner is shown in Figure 9. Figure 10 shows a feeding device for mesuring mono-component toner charges. To measure a mono-component toner with 


\section{Nomenclature}

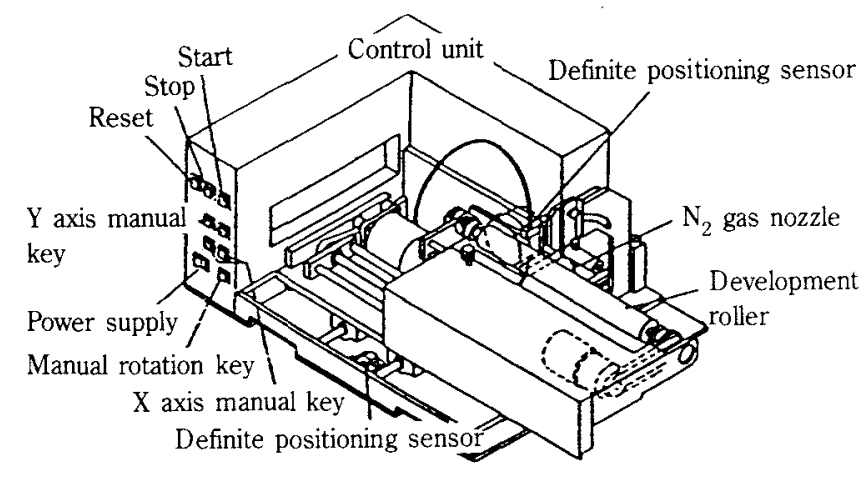

Fig. 10 Feeding device of charged single-component toner

the feeding device, a developing roller with a monocomponent toner affixed to it (i.e.-after the toner has been charged) is removed from the copying machines and set directly onto the feeding device. The toner on the roller surface is fed into the measurement area by a jet of nitrogen gas. The roller is moved into a different position after each jet to ensure that all of the toner particles are blown from the roller surface. The results of measuring two different mono-component toners show that the measurements are substantially identical in terms of the distribution of particle size, but different in the distribution of particle charge. It is assumed that these different measurements reflect two samples having different compositions of the charge control agent and the like, althrough the particle sizes are similar to each other.

\section{Conclusion}

We have presented a method for simultaneously measuring the size and charge of toner particles by observing their velocity and oscillation in acoustic and electrical fields using the Laser Doppler method. In addition, we showed examples which evaluated the relationship between the particle size and the electrostatic charge of toners by measuring the toners using this Laser Doppler method.

Now we are making efforts to improve the accuracy and reproducibility of the measurement through further modifications in order to develop an apparatus that is easier to operate, and capable more accurate measurements, even at a production site.
$C_{m}[-] \quad$ Cunningham's correction factor

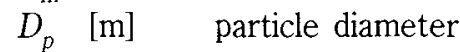

$E \quad[\mathrm{~V} / \mathrm{m}] \quad$ electrical field strength

$L \quad[\mathrm{~m}] \quad$ distance between parallel electrode plates

$m_{p} \quad[\mathrm{~kg}] \quad$ mass of particle

$q \quad[\mathrm{C}] \quad$ charge of particle

$\begin{array}{ll}u_{g} & {[\mathrm{~m}] \quad \text { amplitude of sound wave }}\end{array}$

$\begin{array}{ll}V & {[\mathrm{~V}] \quad \text { applied voltage }}\end{array}$

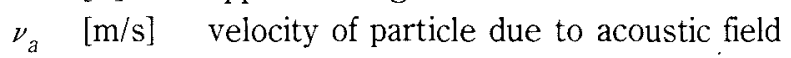

$\nu_{e}[\mathrm{~m} / \mathrm{s}]$ velocity of particle due to electric field

$\nu_{p} \quad[\mathrm{~m} / \mathrm{s}] \quad$ velocity of particle

$\eta \quad[\mathrm{Pa} \cdot \mathrm{s}] \quad$ viscosity of air

$\theta \quad$ [rad] half crossing angle of laser beams

$\begin{array}{ll}\lambda & {[\mathrm{m}] \quad \text { wave length of laser }}\end{array}$

$\rho_{p} \quad\left[\mathrm{~kg} / \mathrm{m}^{3}\right]$ density of particle

$\begin{array}{lll}\tau_{p} & {[\mathrm{~s}] \quad \text { relaxation time of particle }}\end{array}$

$\phi \quad$ [rad] angle of phase lag

$\psi$ [rad] inclination of movement of particle to crossing beam

$\omega \quad[\mathrm{rad} / \mathrm{s}]$ angular velocity of sound

$\begin{array}{ll}\nabla_{f} & {[\mathrm{~Hz}] \quad \text { beat frequency }}\end{array}$

\section{Literature cited}

1) Mazumder, M. K., et al.: IEEE Ind. Appl. Soc. Annual meeting, Part II p. 1606 (1987).

2) Yokoyama, T.et al.: Outline of Presentations at the 25th Symposium on Fine Particles, p. 82, Sapporo (1987)

3) Yatagai, T.: Applied Optics; Guide to Optical Instrumentation, p. 164, Maruzen (1988)

4) Kitabatake, Y.: Funsai, No. 32, p.86 (1988)

5) Tsujimoto, H., et al.: IS \& T's 7th International Congress on Advances in Non-Impact Printing Technologies, 1, p.401 (1991).

6) Kutsuwada, N. et al.: IS \& T's 7th International Congress on Advances in Non-Impact Printing Technologies, 2, p.84 (1991). 